\title{
Sarcopenia Is Associated with Mortality in Adults: A Systematic Review and Meta-Analysis
}

\author{
Jane $\mathrm{Xu}^{\mathrm{a}}$ Ching S. Wan ${ }^{\mathrm{a}, \mathrm{b}}$ Kiriakos Ktoris ${ }^{\mathrm{c}}$ Esmee M. Reijnierse ${ }^{\mathrm{a}, \mathrm{d}}$ \\ Andrea B. Maier ${ }^{a, c, e, f}$ \\ aDepartment of Medicine and Aged Care, @AgeMelbourne, The Royal Melbourne Hospital, The University of \\ Melbourne, Parkville, VIC, Australia; 'bursing Research Institute, St Vincent's Health Network Sydney, St Vincent's \\ Hospital Melbourne and Australian Catholic University, Melbourne, VIC, Australia; 'Department of Human \\ Movement Sciences, @AgeAmsterdam, Vrije Universiteit Amsterdam, Amsterdam Movement Sciences, Amsterdam, \\ The Netherlands; 'Department of Rehabilitation Medicine, Amsterdam UMC, Vrije Universiteit Amsterdam, \\ Amsterdam Movement Sciences, Amsterdam, The Netherlands; ${ }^{e}$ Healthy Longevity Program, Yong Loo Lin School \\ of Medicine, National University of Singapore, Singapore, Singapore; 'Centre for Healthy Longevity, @AgeSingapore, \\ National University Health System, Singapore, Singapore
}

\section{Keywords}

Sarcopenia $\cdot$ Muscular atrophy $\cdot$ Mortality $\cdot$ Population groups

\begin{abstract}
Background: Sarcopenia can predispose individuals to falls, fractures, hospitalization, and mortality. The prevalence of sarcopenia depends on the population studied and the definition used for the diagnosis. Objective: This systematic review and meta-analysis aimed to investigate the association between sarcopenia and mortality and if it is dependent on the population and sarcopenia definition. Methods: A systematic search was conducted in MEDLINE, EMBASE, and Cochrane from 1 January 2010 to 6 April 2020 for articles relating to sarcopenia and mortality. Articles were included if they met the following criteria - cohorts with a mean or median age $\geq 18$ years and either of the following sarcopenia definitions: Asian Working Group for Sarcopenia (AWGS and AWGS2019), European Working Group on Sarcopenia in Older People (EWGSOP and EWGSOP2), Foundation for the National Institutes of Health (FNIH), International Working
\end{abstract}

Group for Sarcopenia (IWGS), or Sarcopenia Definition and Outcomes Consortium (SDOC). Hazard ratios (HR) and odds ratios (OR) were pooled separately in meta-analyses using a random-effects model, stratified by population (community-dwelling adults, outpatients, inpatients, and nursing home residents). Subgroup analyses were performed for sarcopenia definition and follow-up period. Results: Out of 3,025 articles, 57 articles were included in the systematic review and 56 in the meta-analysis $(42,108$ participants, mean age of $49.4 \pm 11.7$ to $86.6 \pm 1.0$ years, $40.3 \%$ females). Overall, sarcopenia was associated with a significantly higher risk of mortality (HR: 2.00 [95\% Cl: 1.71, 2.34]; OR: 2.35 [95\% Cl: 1.64, 3.37]), which was independent of population, sarcopenia definition, and follow-up period in subgroup analyses. Conclusions: Sarcopenia is associated with a significantly higher risk of mortality, independent of population and sarcopenia definition, which highlights the need for screening and early diagnosis in all populations.

(C) 2021 The Author(s) Published by S. Karger AG, Basel Jane Xu and Ching S. Wan contributed equally.
(C) 2021 The Author(s)

Published by S. Karger AG, Basel

This is an Open Access article licensed under the Creative Common Attribution-NonCommercial-4.0 International License (CC BY-NC) (http://www.karger.com/Services/OpenAccessLicense), applicable to the online version of the article only. Usage and distribution for commercial purposes requires written permission.
Correspondence to:

Andrea B. Maier, a.b.maier@vu.nl 


\section{Introduction}

Sarcopenia, age-related low muscle mass and function, is prevalent in $9.9-40.4 \%$ of community-dwelling adults $[1,2], 2-34 \%$ of outpatients [3], and $56 \%$ of hospitalized patients [4]. Sarcopenia is highly prevalent as comorbid disease, for example, in individuals with cardiovascular disease, dementia, diabetes mellitus, and respiratory disease [5]. Sarcopenia definitions have been proposed by various working groups and include muscle mass, muscle strength, and physical performance combinations and vary in cutoff points and diagnostic algorithms [6-11]. Independent of the definition used, sarcopenia is associated with adverse health outcomes such as falls and fractures [12], functional decline [13], and hospitalization [14].

Sarcopenia is associated with a 2 times higher risk of mortality in community-dwelling adults [15] and nursing home residents [16] and 3 times higher risk in cancer patients [17]. Previous systematic reviews evaluating the association of sarcopenia and mortality included articles published until 2017 [14-16, 18]. As new definitions of sarcopenia were proposed in 2018 [7], 2019 [6], and 2020 [19] and the prevalence of sarcopenia depends on the studied population and the definition used [20,21], an updated systematic review on the association between sarcopenia and mortality is needed. The aim of this systematic review and meta-analysis was to assess the association between sarcopenia and mortality and if this association is dependent on population, sarcopenia definition, and follow-up period.

\section{Methods}

Data Sources and Searches

The Preferred Reporting Items for Systematic Review and Meta-Analysis (PRISMA) was followed for all steps in this systematic review (see online suppl. Table 1; for all online suppl. material, see www.karger.com/doi/10.1159/000517099) [22]. The protocol was registered on PROSPERO (international prospective register of systematic reviews): CRD42020179744. The electronic databases MEDLINE, EMBASE, and Cochrane Library (CENTRAL) were searched for from 1 January 2010 until 6 April 2020 for articles relating to sarcopenia and mortality. The start date of the search was chosen as 2010, the year the first working group definition was published [11]. The search was developed with the assistance of a senior academic librarian from a biomedical university library. The search strategy and search terms used for this search are detailed in online suppl. Table 2. The reference list of each included article was manually searched to identify additional articles. Authors were contacted if additional information was required to include the article in the meta-analysis.

\section{Article Selection}

Two reviewers independently screened the titles and abstracts and subsequently the included full text of articles (J.X. and K.K.). Any discrepancies were resolved by a third reviewer (C.S.W.). Articles were included if they met the following criteria - a longitudinal cohort with a mean or median age $\geq 18$ years of age and reporting the association between sarcopenia and mortality using one of the following sarcopenia definitions: Asian Working Group for Sarcopenia (AWGS and AWGS2019) [6, 9], European Working Group on Sarcopenia in Older People (EWGSOP and EWGSOP2) $[7,11]$, Foundation for the National Institutes of Health (FNIH) [8], International Working Group for Sarcopenia (IWGS) [10], or Sarcopenia Definition and Outcomes Consortium (SDOC) [19]. Exclusion criteria included case reports ( $<20$ individuals), reviews, conference abstracts, articles that were not published in the English language, or full text was not available. If articles reported data of the same cohort [23-26], the article with the largest sample size was included $[24,26]$.

\section{Data Extraction and Risk of Bias Assessment}

The following data were extracted independently by 2 reviewers (J.X. and K.K.): first author, publication year, country of included participants, sample size, sex, age, population, sarcopenia definition, sarcopenia prevalence, methodologies to measure muscle mass, muscle strength and physical performance and the respective cutoff values used, follow-up period, effect size and its $95 \%$ confidence intervals (CI) of the association between sarcopenia and mortality, and any adjustments made if multivariable models were reported. The weighted mean for age was calculated if age was stratified by groups.

The risk of bias assessment was performed independently by 2 reviewers (J.X. and K.K.) using a modified Newcastle-Ottawa Scale (NOS) [27] provided in online suppl. Table 3. Any discrepancies were resolved by a third reviewer (C.S.W.). The highest possible score for NOS, reflecting the lowest risk of bias, was 9 stars. A median score of 7 was used as the cutoff to classify an article as having either a low or high risk of bias [27].

\section{Data Synthesis and Statistical Analysis}

A random-effects model was used to pool hazard ratio (HR) and odds ratio (OR) separately for the association between sarcopenia and mortality. All analyses were stratified by population (community-dwelling adults, outpatients, inpatients, and nursing home residents). For the main meta-analysis, if multiple sarcopenia definitions were used, the following sarcopenia definition was included in the primary analysis for the association between sarcopenia and mortality: (1) the definition that was developed across the cohort's country was selected (i.e., EWGSOP for European cohort) and (2) if the same definition was used more than once, the definition with the cutoff points closest to the original cutoff points was included.

If more than 1 statistical adjustment model for the association between sarcopenia and mortality was reported, the model included in the meta-analyses was based on the following hierarchy: (1) age and sex (when stratified by sex, the model that adjusted only for age was included; when stratified by age, the model that adjusted only for sex was included); (2) age, sex, cognitive impairment, and/or other comorbidities; (3) age, sex, cognitive impairment and/or other comorbidities, and other confounders; (4) age and other confounders; (5) age alone; and (6) 


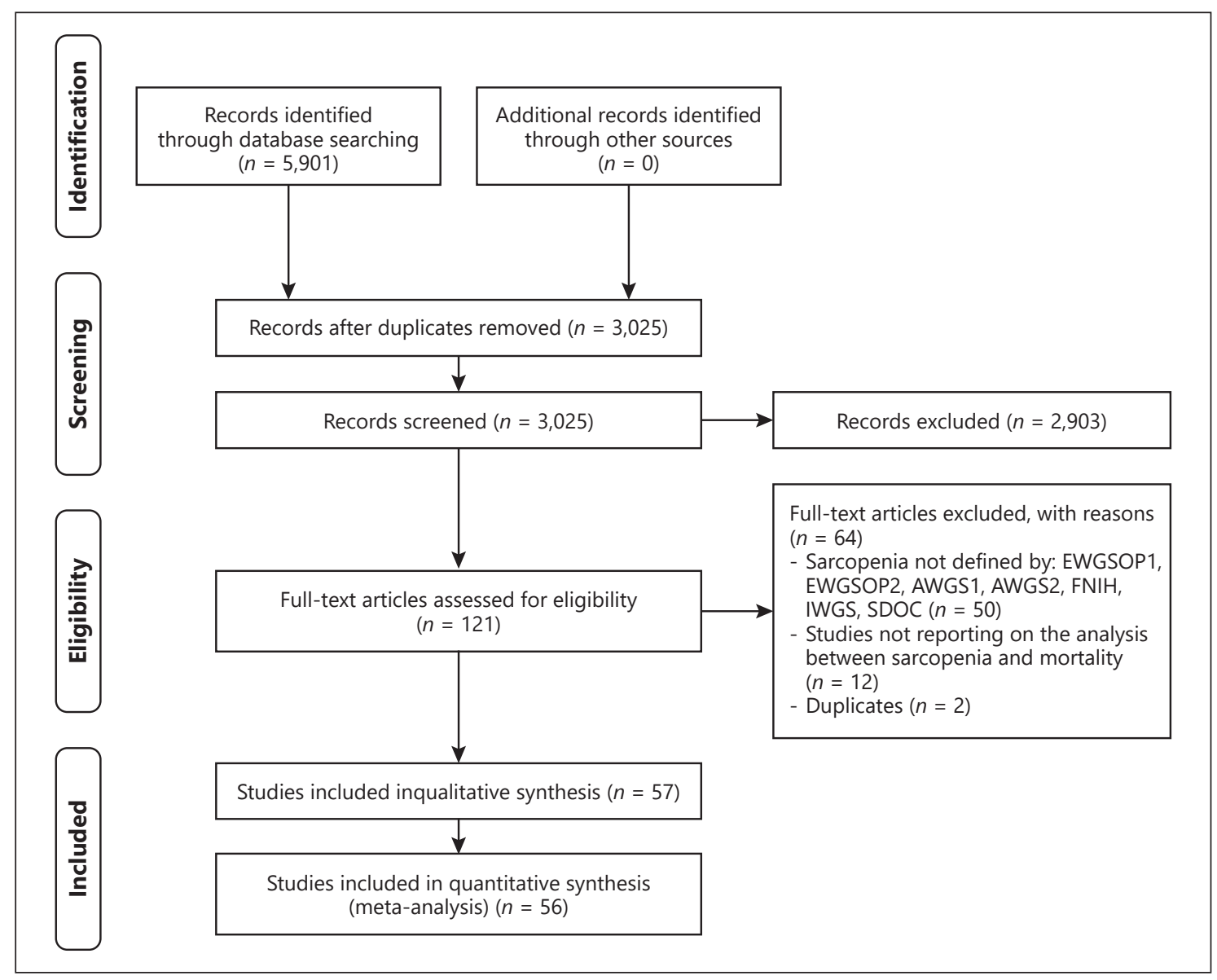

Fig. 1. PRISMA flow diagram of the article selection. PRISMA, Preferred Reporting Items for Systematic Review and Meta-Analysis.

crude model. When articles reported more than 1 follow-up period, the model with the shortest follow-up time was included in the meta-analysis as confounding factors may have a greater effect at longer follow-up periods. Subgroup analyses for sarcopenia definition, follow-up period, and risk of bias were performed if 2 or more articles were included. For all populations, the median follow-up period was used as the cutoff for short $(<$ median) and long term ( $\geq$ median).

Heterogeneity was assessed with $I^{2}$ statistics for each subgroup, with low defined as $I^{2} \leq 25 \%$, moderate as $I^{2}=25-75 \%$, and high as $\geq 75 \%$ [28]. The Cochran's $Q$ value was used to evaluate betweengroup heterogeneity and $p$ value of $<0.05$ of the $Q$ value $\left(Q_{b}\right)$ indicated a statistically significant difference between the groups [28]. Publication bias of the overall association of sarcopenia with mortality was assessed by funnel plots of log $\mathrm{HR}$ and $\log \mathrm{OR}$ against its standard error. Egger's regression test was used to evaluate the statistical significance of publication bias [29]. $p$ values $<0.05$ were considered statistically significant (2-tailed). Meta-analysis was performed using Comprehensive Meta-Analysis (CMA version 3.3; Biostat Inc., Englewood, NJ, USA).

Sarcopenia and Mortality

\section{Results}

After retrieval of 5,901 articles from electronic databases and removal of duplicates, 3,025 articles were identified for title and abstract screening. In total, 121 articles were screened for full text, of which 57 articles were included in this systematic review. The authors of 1 article did not provide additional information for the meta-analysis; therefore, 56 articles were included in the meta-analysis (shown in Fig. 1).

Table 1 shows the study characteristics of the included articles. Nineteen articles included community-dwelling cohorts (31,008 individuals, age range of $\geq 60$ years to 86.6 \pm 1.0 years, $36.6 \%$ females) and the EWGSOP was most used (12/19 articles) [26, 30-40], followed by FNIH (10/19 articles) $[33,34,37-39,41-45]$, AWGS (4/19 articles) [34, 37, 44, 46], IWGS (3/19 articles) [33, 34, 37], and EWGSOP2 (3/19 articles) [39, 40, 47]. Nine articles 


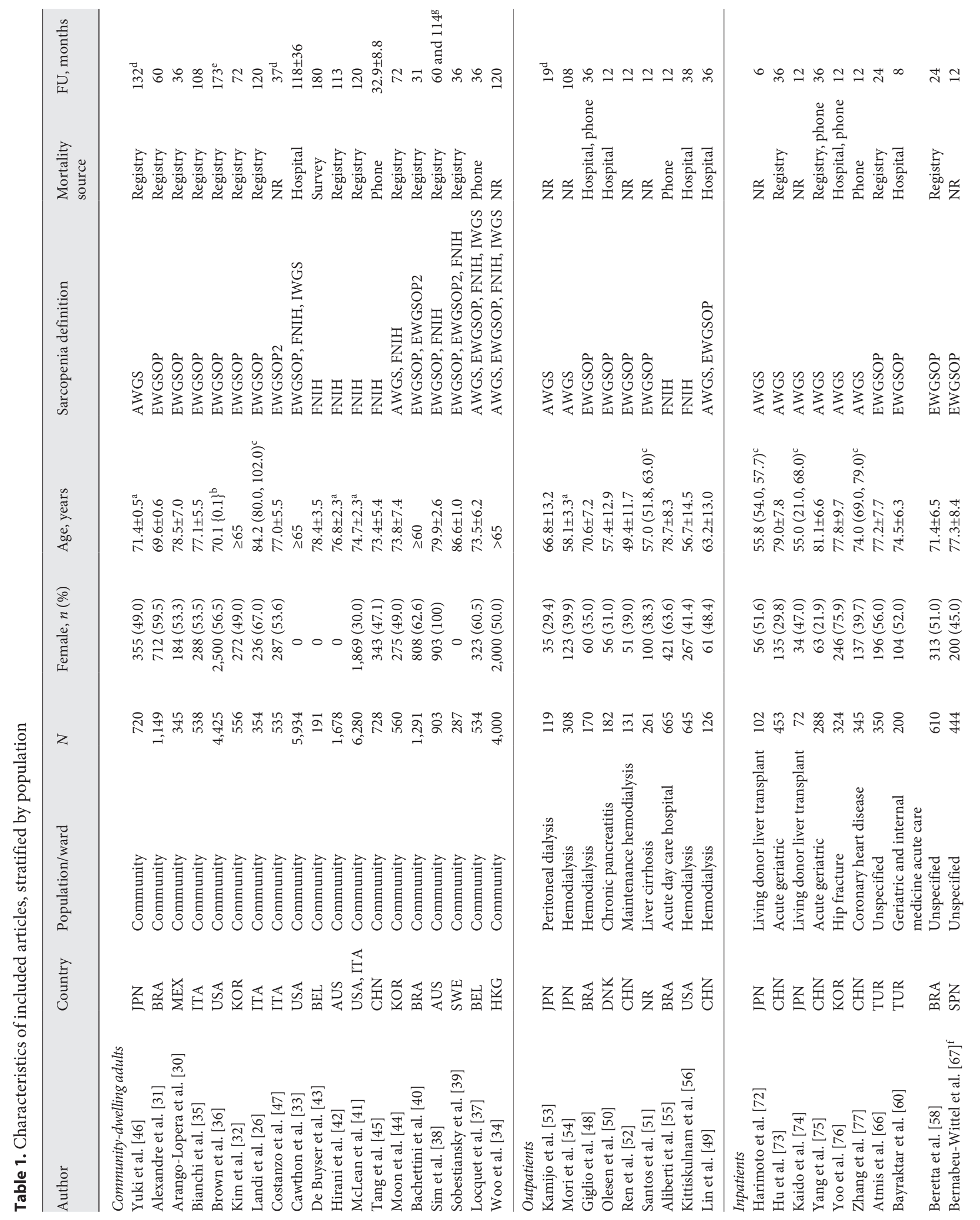




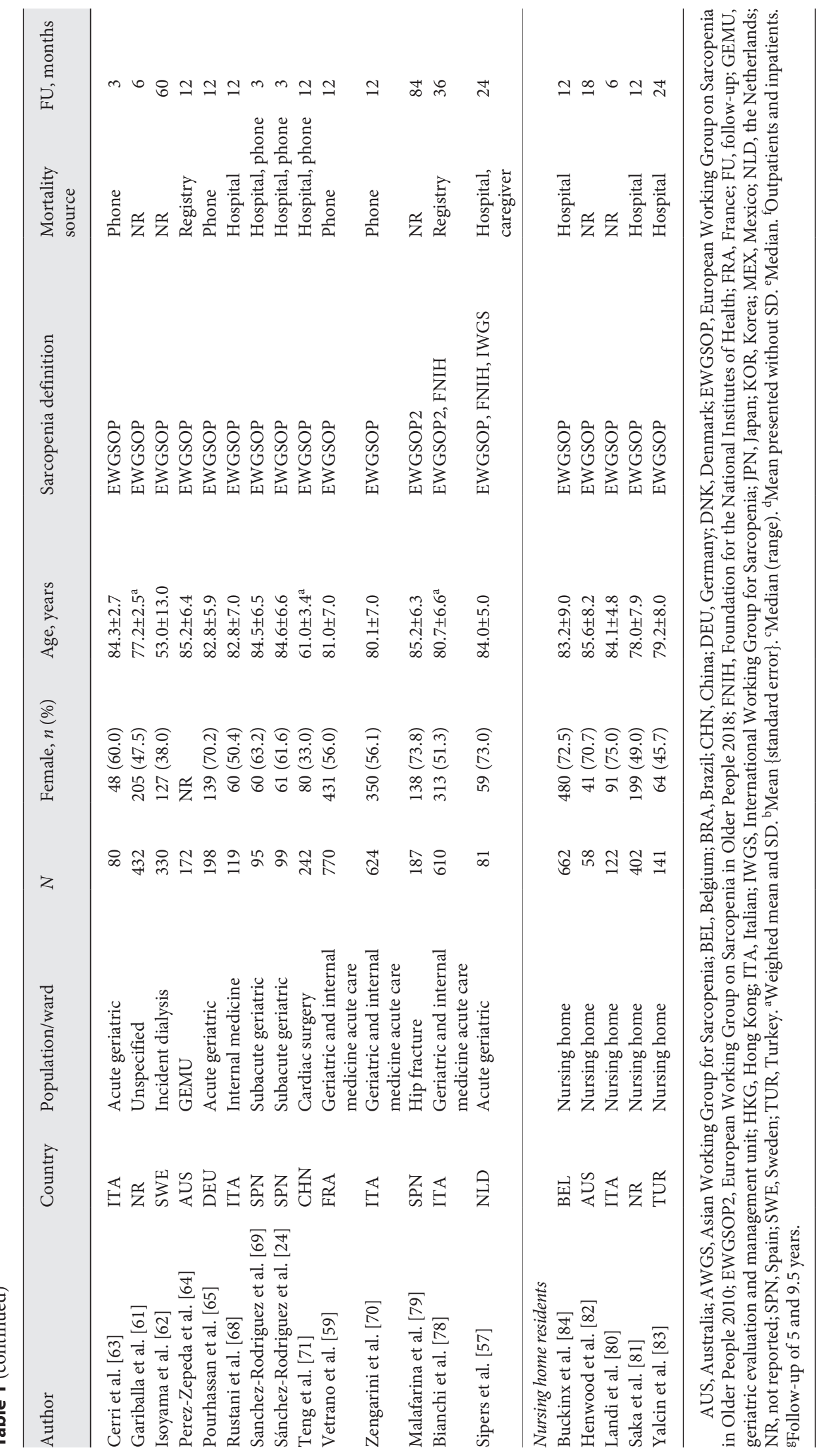


included outpatient cohorts (2,607 individuals, mean age $49.4 \pm 11.7$ to $78.7 \pm 8.3$ years, $45.0 \%$ females $)$ and the EWGSOP was most used (5/9 articles) [48-52], followed by AWGS (3/9 articles) $[49,53,54]$ and FNIH (2/9 articles) $[55,56]$. Twenty-four articles included inpatient cohorts $(7,227$ individuals, median age of 55.0 [21.0, 68.0] to mean age of $85.2 \pm 6.4$ years, $49.2 \%$ females) and the EWGSOP was most used (16/24 articles) [24, 57-71], followed by AWGS (6/24 articles) [72-77], EWGSOP2 (2/24 articles) [78, 79], FNIH (2/24 articles) [57, 78], and IWGS (1/24 articles) [57]. Five articles included nursing home cohorts $(1,385$ individuals, mean age of 78.0.9 \pm 7.9 to $85.6 \pm 8.2$ years, $63.2 \%$ females), and all used the EWGSOP definition [80-84]. The measurement methods and cutoffs for each sarcopenia definition used are given in online suppl. Table 4 . The follow-up period ranged from 31 to 180 months for community-dwelling adults, $12-108$ months for outpatients, 3-84 months for inpatients, and 6-24 months for nursing home residents. Short-term follow-up was defined as $<72$ months for community-dwelling adults, $<36$ months for outpatients, and $<24$ months for inpatients.

\section{Risk of Bias}

Table 2 shows the individual NOS scores for each criterion of the included articles. The risk of bias assessment resulted in 40 articles with low risk of bias (17 in community-dwelling adults, 6 in outpatients, 14 in inpatients, and 3 in nursing home residents) and 17 as high risk of bias ( 2 in community-dwelling adults, 3 in outpatients, 10 in inpatients, and 2 in nursing home residents).

\section{Meta-Analysis}

Table 3 shows the HRs and ORs of the association between sarcopenia and mortality that were included in the meta-analyses, stratified by population. All reported statistical models of the included articles can be found in online suppl. Table 5. Overall, sarcopenia was statistically significantly associated with a higher risk of mortality $\left(\mathrm{HR}=2.00\right.$ [95\% CI: 1.71, 2.34], $I^{2}: 46.9 \% ; \mathrm{OR}=2.35$ [95\% CI: 1.64, 3.37], $I^{2}: 43.7 \%$ ) (shown in Fig. 2, 3). The association was independent of population: communitydwelling adults ( $\mathrm{HR}=1.88$ [95\% CI: 1.59, 2.25], $I^{2}: 32.4 \%$; $\mathrm{OR}=1.98$ [95\% CI: 1.03, 3.79], $\left.I^{2}: 0 \%\right)$, outpatients (HR $=1.81$ [95\% CI: 1.28, 2.55], $I^{2}: 12.4 \%$; OR $=4.33$ [95\% CI: $\left.1.25,14.9], I^{2}: 17.4 \%\right)$, inpatients ( $\mathrm{HR}=2.15$ [95\% CI: $1.76,2.62$ ], $I^{2}: 62.1 \%$; OR $=2.62$ [95\% CI: 1.72, 4.99], $I^{2}$ : $60.3 \%$ ), and nursing home residents ( $\mathrm{HR}=2.84$ [95\% CI: $1.40,5.73$ ] $I^{2}: 0 \%$; OR $=1.90$ [95\% CI: 1.01, 3.57], $I^{2}$ : $0.68 \%$ ) (shown in Fig. 2,3 ). There was no statistically sig- nificant difference between the heterogeneity of populations (HR: $Q_{b} p=0.528$; OR: $Q_{b} p=0.594$ ).

Online suppl. Figures 1-4 show the subgroup analyses of the association stratified by sarcopenia definition. Sarcopenia diagnosed by the EWGSOP, EWGSOP2, and FNIH was associated with significantly higher risk of mortality in all populations: community-dwelling adults (EWGSOP: HR $=1.90$ [95\% CI: 1.52, 2.37], $I^{2}: 50.4 \%$; EWGSOP2: $\mathrm{HR}=1.73$ [95\% CI: 1.02, 2.93], $I^{2}: 0 \%$; FNIH: HR $=1.80$ [95\% CI: 1.41, 2.29], $I^{2}: 5.4 \%$ ), outpatients (EWGSOP: HR $=2.37$ [95\% CI: 1.43, 3.93], $I^{2}$ : 29.8\%; FNIH: HR = 1.69 [95\% CI: 1.16, 2.47], $I^{2}: 0 \%$ ), and inpatients (EWGSOP: $\mathrm{HR}=1.94$ [95\% CI: 1.39, 2.71], $I^{2}: 45.3 \%$; OR $=2.34$ [95\% CI: 1.37, 4.00], $I^{2}: 60.4 \%$; FNIH: HR $=2.16$ [95\% CI: $\left.1.19,3.93], I^{2}: 81.3 \%\right)$. Sarcopenia diagnosed by the AWGS was associated with significantly higher risk of mortality in community-dwelling adults (AWGS: HR = 1.96 [95\% CI: 1.29, 2.96], $I^{2}$ : 56.7\%) and inpatients (AWGS: $\mathrm{HR}=2.31$ [95\% CI: 1.47, 3.63], $I^{2}: 66.9 \%$; OR $=6.41$ [95\% CI: 1.76, 23.28], $I^{2}: 17.6$ ) but not significant in outpatients (HR: 1.40 [95\% CI: $\left.0.91,2.16], I^{2}: 0 \%\right)$. There was no significant difference between the heterogeneity of effect estimates (community-dwelling adults [HR: $Q_{b} p=0.972$ ], outpatients [HR: $\left.Q_{b} p=0.300\right]$, and inpatients [HR: $Q_{b} p=0.883$; OR: $Q_{b} p$ $=0.158]$ ).

The significant association between sarcopenia and mortality was independent of the follow-up period in all populations: community-dwelling adults (long-term HR $=1.78$ [95\% CI: 1.48, 2.14], $I^{2}: 36.7 \%$; short-term HR = 2.01 [95\% CI: 1.55, 2.60], $I^{2}: 0 \%$ ), outpatients (long-term $\mathrm{HR}=1.64$ [95\% CI: 1.12, 2.38], $I^{2}: 0 \%$; short-term HR = 2.12 [95\% CI: 1.22, 3.70], $I^{2}: 73.0 \%$ ), and inpatients (longterm $\mathrm{HR}=2.68$ [95\% CI: 2.02, 3.55], $I^{2}: 58.3 \%$; short-term $\mathrm{HR}=1.51$ [95\% CI: 1.06, 2.17], $\left.I^{2}: 32.5 \%\right)$. There was no statistically significant difference between the heterogeneity of effect estimates for the follow-up period for community-dwelling adults $\left(\mathrm{HR}: Q_{b} p=0.461\right)$ and outpatients (HR: $\left.Q_{b} p=0.448\right)$, but for inpatients (HR: $Q_{b} p=$ 0.015) (online suppl. Fig. 5-7).

The association of sarcopenia with mortality was independent of risk of bias (high risk of bias: $\mathrm{HR}=2.58[95 \%$ CI: 1.90, 3.52], $I^{2}: 63.7 \%$; OR $=3.19$ [95\% CI: $2.23,4.56$ ], $I^{2}: 20.1 \%$; low risk of bias: $\mathrm{HR}=1.89$ [95\% CI: 1.66, 2.15], $I^{2}: 36.9 \% ;$ OR $=1.74$ [95\% CI: $\left.\left.1.29,2.34\right], I^{2}: 32.2 \%\right)$. The heterogeneity of effect estimates for risk of bias was not statistically significant for HRs $\left(Q_{b} p=0.069\right)$, but for ORs $\left(Q_{b} p=0.010\right)$ (online suppl. Fig. 8, 9). Overall, heterogeneity was low to moderate across all pooled HRs and ORs apart from the pooled FNIH HR stratifying for sarcope- 
Table 2. Quality assessment of included articles using the NOS, stratified by population

\begin{tabular}{|c|c|c|c|c|c|c|c|c|c|}
\hline \multirow[t]{2}{*}{ Author } & \multicolumn{4}{|c|}{ Selection } & \multirow{2}{*}{$\begin{array}{l}\text { Compa- } \\
\text { rability } \\
\text { Q1 }\end{array}$} & \multicolumn{3}{|c|}{ Outcome } & \multirow{2}{*}{$\begin{array}{l}\text { Total } \\
\text { score }\end{array}$} \\
\hline & Q1 & Q2 & Q3 & Q4 & & Q1 & Q2 & Q3 & \\
\hline \multicolumn{10}{|l|}{ Community-dwelling adults } \\
\hline Yuki et al. [46] & 1 & 1 & 1 & 1 & 1 & 1 & 1 & 1 & 8 \\
\hline Alexandre et al. [31] & 0 & 1 & 1 & 1 & 2 & 1 & 1 & 1 & 8 \\
\hline Arango-Lopera et al. [30] & 0 & 0 & 1 & 1 & 1 & 0 & 1 & 1 & 5 \\
\hline Bianchi et al. [35] & 0 & 1 & 1 & 1 & 2 & 1 & 1 & 1 & 8 \\
\hline Brown et al. [36] & 0 & 1 & 1 & 1 & 2 & 1 & 1 & 0 & 7 \\
\hline Kim et al. [32] & 0 & 1 & 1 & 1 & 1 & 1 & 1 & 0 & 6 \\
\hline Landi et al. [26] & 0 & 1 & 1 & 1 & 2 & 1 & 1 & 1 & 8 \\
\hline Costanzo et al. [47] & 0 & 1 & 1 & 1 & 2 & 0 & 1 & 1 & 7 \\
\hline Cawthon et al. [33] & 1 & 1 & 1 & 1 & 1 & 1 & 1 & 1 & 8 \\
\hline De Buyser et al. [43] & 1 & 0 & 1 & 1 & 1 & 1 & 1 & 1 & 7 \\
\hline Hirani et al. [42] & 1 & 1 & 1 & 1 & 2 & 0 & 1 & 1 & 8 \\
\hline McLean et al. [41] & 1 & 1 & 1 & 1 & 1 & 1 & 1 & 0 & 7 \\
\hline Tang et al. [45] & 1 & 1 & 1 & 1 & 2 & 1 & 1 & 1 & 9 \\
\hline Moon et al. [44] & 1 & 0 & 1 & 1 & 1 & 1 & 1 & 1 & 7 \\
\hline Bachettini et al. [40] & 0 & 1 & 1 & 1 & 2 & 0 & 1 & 1 & 7 \\
\hline Sim et al. [38] & 1 & 1 & 1 & 1 & 1 & 1 & 1 & 1 & 8 \\
\hline Sobestiansky et al. [39] & 1 & 1 & 1 & 1 & 1 & 1 & 1 & 1 & 8 \\
\hline Locquet et al. [37] & 0 & 1 & 1 & 1 & 2 & 1 & 1 & 1 & 8 \\
\hline Woo et al. [34] & 0 & 1 & 1 & 1 & 1 & 0 & 1 & 1 & 7 \\
\hline \multicolumn{10}{|l|}{ Outpatients } \\
\hline Kamijo et al. [53] & 1 & 1 & 1 & 1 & 2 & 0 & 1 & 1 & 8 \\
\hline Mori et al. [54] & 1 & 1 & 1 & 1 & 2 & 0 & 1 & 0 & 7 \\
\hline Giglio et al. [48] & 1 & 1 & 1 & 1 & 2 & 1 & 1 & 1 & 9 \\
\hline Olesen et al. [50] & 0 & 1 & 1 & 1 & 0 & 0 & 1 & 1 & 5 \\
\hline Ren et al. [52] & 0 & 1 & 1 & 1 & 0 & 0 & 1 & 1 & 5 \\
\hline Santos et al. [51] & 1 & 1 & 1 & 1 & 0 & 0 & 1 & 1 & 6 \\
\hline Aliberti et al. [55] & 0 & 1 & 1 & 1 & 2 & 1 & 1 & 1 & 8 \\
\hline Kittiskulnam et al. [56] & 0 & 1 & 1 & 1 & 2 & 1 & 1 & 1 & 8 \\
\hline Lin et al. [49] & 0 & 1 & 1 & 1 & 2 & 1 & 1 & 1 & 8 \\
\hline \multicolumn{10}{|l|}{ Inpatients } \\
\hline Harimoto et al. [72] & 0 & 1 & 1 & 1 & 2 & 0 & 1 & 1 & 7 \\
\hline Hu et al. [73] & 0 & 1 & 1 & 1 & 0 & 1 & 1 & 1 & 6 \\
\hline Kaido et al. [74] & 1 & 1 & 1 & 1 & 0 & 0 & 1 & 1 & 6 \\
\hline Yang et al. [75] & 0 & 1 & 1 & 1 & 2 & 1 & 1 & 1 & 8 \\
\hline Yoo et al. [76] & 1 & 1 & 1 & 1 & 2 & 1 & 1 & 1 & 9 \\
\hline Zhang et al. [77] & 1 & 1 & 1 & 1 & 2 & 1 & 1 & 1 & 9 \\
\hline Atmis et al. [66] & 0 & 1 & 1 & 1 & 2 & 1 & 1 & 0 & 7 \\
\hline Bayraktar et al. [60] & 0 & 1 & 1 & 1 & 0 & 0 & 1 & 1 & 5 \\
\hline Beretta et al. [58] & 0 & 1 & 1 & 1 & 2 & 1 & 1 & 0 & 7 \\
\hline Bernabeu-Wittel et al. [67] ${ }^{\mathrm{a}}$ & 0 & 1 & 1 & 1 & 2 & 0 & 1 & 0 & 6 \\
\hline Cerri et al. [63] & 0 & 1 & 1 & 1 & 0 & 1 & 1 & 1 & 6 \\
\hline Gariballa et al. [61] & 0 & 0 & 1 & 1 & 0 & 0 & 1 & 1 & 4 \\
\hline Isoyama et al. [62] & 0 & 1 & 1 & 1 & 2 & 0 & 1 & 0 & 6 \\
\hline Perez-Zepeda et al. [64] & 0 & 1 & 1 & 1 & 2 & 1 & 1 & 1 & 8 \\
\hline Pourhassan et al. [65] & 0 & 1 & 1 & 1 & 2 & 1 & 1 & 0 & 7 \\
\hline Rustani et al. [68] & 0 & 1 & 1 & 1 & 0 & 1 & 1 & 1 & 6 \\
\hline Sanchez-Rodriguez et al. [69] & 0 & 1 & 1 & 1 & 2 & 1 & 1 & 1 & 8 \\
\hline Sánchez-Rodriguez et al. [24] & 0 & 1 & 1 & 1 & 0 & 1 & 1 & 1 & 6 \\
\hline Teng et al. [71] & 0 & 1 & 1 & 1 & 0 & 1 & 1 & 1 & 6 \\
\hline Vetrano et al. [59] & 0 & 1 & 1 & 1 & 2 & 1 & 1 & 1 & 8 \\
\hline Zengarini et al. [70] & 0 & 1 & 1 & 1 & 2 & 1 & 1 & 1 & 8 \\
\hline
\end{tabular}


Table 2 (continued)

\begin{tabular}{|c|c|c|c|c|c|c|c|c|c|}
\hline \multirow[t]{2}{*}{ Author } & \multicolumn{4}{|c|}{ Selection } & \multirow{2}{*}{$\begin{array}{l}\text { Compa- } \\
\text { rability } \\
\text { Q1 }\end{array}$} & \multicolumn{3}{|c|}{ Outcome } & \multirow{2}{*}{$\begin{array}{l}\text { Total } \\
\text { score }\end{array}$} \\
\hline & Q1 & Q2 & Q3 & $\mathrm{Q} 4$ & & Q1 & Q2 & Q3 & \\
\hline Malafarina et al. [79] & 0 & 1 & 1 & 1 & 2 & 0 & 1 & 1 & 7 \\
\hline Bianchi et al. [78] & 1 & 1 & 1 & 1 & 2 & 1 & 1 & 1 & 9 \\
\hline Sipers et al. [57] & 1 & 1 & 1 & 1 & 0 & 1 & 1 & 1 & 7 \\
\hline \multicolumn{10}{|l|}{ Nursing home residents } \\
\hline Buckinx et al. [84] & 0 & 1 & 1 & 1 & 2 & 1 & 1 & 1 & 8 \\
\hline Henwood et al. [82] & 0 & 0 & 1 & 1 & 2 & 0 & 1 & 0 & 5 \\
\hline Landi et al. [80] & 0 & 1 & 1 & 1 & 2 & 1 & 1 & 1 & 8 \\
\hline Saka et al. [81] & 0 & 1 & 1 & 1 & 0 & 0 & 1 & 1 & 5 \\
\hline Yalcin et al. [83] & 0 & 1 & 1 & 1 & 2 & 1 & 1 & 1 & 8 \\
\hline
\end{tabular}

NOS, Newcastle-Ottawa Scale. ${ }^{a}$ Outpatients and inpatients.

nia definitions in inpatients, where heterogeneity was high.

\section{Publication Bias}

Asymmetry was observed by visual inspection of funnel plots for articles that reported HR and OR (online suppl. Fig. 10). Egger's regression test revealed significant publication bias among the included articles in the metaanalysis for articles that reported HRs $(p=0.006)$, but not for articles that reported ORs $(p=0.053)$.

\section{Discussion}

Sarcopenia is significantly associated with mortality in adults, independent of the population studied, sarcopenia definition, follow-up period, and risk of bias. This review adds significantly to the literature, as it includes the updated definition of sarcopenia, which are being implemented into clinical practice [7]. The findings that sarcopenia is significantly associated with mortality are consistent with the reviews published previously [14-16, 18]. The results from the subgroup analyses showing the independence of the association of population [14], followup [14, 15], and risk of bias [14] are also consistent with the reviews that examined these relations.

Original studies and systematic reviews have extensively demonstrated that individuals with sarcopenia are at risk of functional decline [13], frailty [85], decreased mobility [86], falls, fractures [12], and hospitalization [87], which can all contribute to a higher mortality risk.
One of the main mechanisms relating sarcopenia to mortality is falls. Low muscle mass and strength contribute to the impairment of balance [88], which is associated with falls [89]. As osteoporosis and malnutrition are highly prevalent in older adults [90-92], this increases the susceptibility of fractures accompanying falls that can lead to hospitalization. Prolonged inactivity and bed rest during hospitalization could contribute to a decrease in muscle mass and strength [93], leading to functional decline and a greater risk of future falls following hospital discharge and higher incidence of readmissions [75]. Sarcopenia is also associated with a higher length of hospital stay [94] and as hospitalization contributes to loss of muscle mass and strength [93], this perpetuating cycle of functional decline and rehospitalization may contribute to mortality. Early screening and diagnosis of sarcopenia in primary care and hospitals are crucial for the implementation of prevention or intervention programs to alleviate the associated risks of sarcopenia and reduce the healthcare burden and costs.

Irrespective of the definition used for the diagnosis, sarcopenia was associated with a higher risk of mortality. This is remarkable, as the use of different definitions leads to a different prevalence of sarcopenia $[21,95]$ and therewith to comparisons of different proportions of populations determined to be affected. The association between sarcopenia and other clinically relevant outcomes such as falls and fractures [12] remains significant, while using different definitions highlights the strong clinical association of sarcopenia with adverse health outcomes irrespective of the definition used for diagnosis. Therewith, iden- 
Table 3. The association between sarcopenia and mortality, stratified by population

\begin{tabular}{|c|c|c|c|c|}
\hline Author & $\begin{array}{l}\text { Sarcopenia } \\
\text { definition }\end{array}$ & EM & Effect size $(95 \% \mathrm{CI})$ & Adjustments \\
\hline \multicolumn{5}{|l|}{ Community-dwelling adults } \\
\hline Yuki et al. [46] & AWGS & HR & $\begin{array}{l}\text { M: } 1.86(1.03,3.37) \\
\text { F: } 1.03(0.41,2.60)\end{array}$ & Age \\
\hline Alexandre et al. [31] & EWGSOP & HR & $1.72(1.20,2.47)$ & $\begin{array}{l}\text { Age, sex, income, marital status, education, smoking, weekly alcohol intake, } \\
\text { sedentary lifestyle, PAH, DM, lung disease, CVD stroke, cancer, number of } \\
\text { diseases, falls, hospitalization, MMSE, GDS, ADL, and IADL }\end{array}$ \\
\hline Arango-Lopera et al. [30] & EWGSOP & HR & $2.39(1.05,5.43)$ & Age, IHD, health self-perception, and ADL \\
\hline Bianchi et al. [35] & EWGSOP & $\mathrm{HR}$ & $2.12(1.05,4.30)$ & Age and sex \\
\hline Brown et al. [36] & EWGSOP & HR & $1.40(1.25,1.57)$ & Age and sex \\
\hline Costanzo et al. [47] & EWGSOP2 & HR & $2.30(0.85,6.18)$ & Age and sex \\
\hline Cawthon et al. [33] & FNIH & HR & $3.49(2.01,6.05)$ & Age \\
\hline De Buyser et al. [43] & FNIH & $\mathrm{HR}$ & $2.50(1.30,4.79)$ & Age \\
\hline Hirani et al. [42] & FNIH & HR & $1.69(1.17,2.44)$ & $\begin{array}{l}\text { Age, income, living status, BMI, comorbidities, dementia, ADL disability, low } \\
\mathrm{Hb} \text {, polypharmacy, and low albumin }\end{array}$ \\
\hline McLean et al. [41] & FNIH & HR & $\begin{array}{l}\text { M: } 1.27(0.65,2.46)^{\mathrm{a}} \\
\text { M: } 1.51(0.61,3.71)^{\mathrm{b}} \\
\text { F: } 1.15(0.28,4.70)^{\mathrm{b}} \\
\text { F: } 1.65(0.52,5.25)^{\mathrm{c}} \\
\text { F: } 3.62(0.49,26.6)^{\mathrm{d}} \\
\text { F: } 0.60(0.08,4.56)^{\mathrm{e}}\end{array}$ & Age \\
\hline Bachettini et al. [40] & EWGSOP2 & HR & $1.36(0.52,3.57)$ & $\begin{array}{l}\text { Age, sex, marital status, working, smoking, physical activity at leisure, BMI, } \\
\text { comorbidities, and depressive symptoms }\end{array}$ \\
\hline \multirow[t]{2}{*}{ Sim et al. [38] } & EWGSOP & $\mathrm{HR}$ & $1.88(1.24,2.85)$ & Age \\
\hline & FNIH & HR & $1.08(0.56,2.08)$ & Age \\
\hline \multirow[t]{3}{*}{ Sobestiansky et al. [39] } & EWGSOP & HR & $1.95(1.12,3.40)$ & Age, CCI, education, smoking, and MMSE \\
\hline & EWGSOP2 & HR & $1.70(0.94,3.05)$ & Age, CCI, education, smoking, and MMSE \\
\hline & FNIH & HR & $1.65(0.73,3.72)$ & Age, CCI, education, smoking, and MMSE \\
\hline \multirow[t]{3}{*}{ Locquet et al. [37] } & AWGS & HR & $5.85(2.47,13.8)$ & Age and sex \\
\hline & EWGSOP & HR & $4.20(1.74,10.1)$ & Age and sex \\
\hline & FNIH & HR & $2.47(0.68,8.93)$ & Age and sex \\
\hline
\end{tabular}


Table 3 (continued)

\begin{tabular}{|c|c|c|c|c|}
\hline Author & $\begin{array}{l}\text { Sarcopenia } \\
\text { definition }\end{array}$ & EM & Effect size $(95 \% \mathrm{CI})$ & Adjustments \\
\hline \multirow[t]{3}{*}{ Woo et al. [34] } & EWGSOP & OR & $\begin{array}{l}\text { M: } 2.74(1.95,3.85) \\
\text { F: } 1.55(1.03,2.32)\end{array}$ & $\begin{array}{l}\text { Age, education, COPD, DM, hypertension, CVD, current smoker, MMSE, } \\
\text { and depression }\end{array}$ \\
\hline & FNIH & OR & $\begin{array}{l}\text { M: } 2.32(1.23,4.37) \\
\text { F: } 2.67(1.16,6.15)\end{array}$ & $\begin{array}{l}\text { Age, education, COPD, DM, hypertension, CVD, current smoker, MMSE, } \\
\text { and depression }\end{array}$ \\
\hline & IWGS & OR & $\begin{array}{l}\text { M: } 1.26(0.97,1.63) \\
\text { F: } 1.11(0.81,1.54)\end{array}$ & $\begin{array}{l}\text { Age, education, COPD, DM, hypertension, CVD, current smoker, MMSE, } \\
\text { and depression }\end{array}$ \\
\hline \multicolumn{5}{|l|}{ Outpatients } \\
\hline Mori et al. [54] & AWGS & HR & $1.31(0.81,2.10)$ & $\begin{array}{l}\text { Age, sex, duration of hemodialysis (years), BMI, DM, serum albumin, Kt/V, } \\
\text { and nPCR }\end{array}$ \\
\hline Giglio et al. [48] & EWGSOP & HR & $2.09(1.05,4.20)$ & Age, sex, dialysis vintage, and DM \\
\hline Olesen et al. [50] & EWGSOP & HR & $6.69(1.79,24.9)$ & Crude \\
\hline Ren et al. [52] & EWGSOP & OR & $14.0^{\mathrm{f}}$ & Crude \\
\hline Santos et al. [51] & EWGSOP & OR & $3.06^{\mathrm{f}}$ & Crude \\
\hline Aliberti et al. [55] & FNIH & HR & $1.69(1.05,2.73)$ & $\begin{array}{l}\text { Age, sex, race, income, CCI, depressive symptoms, cognitive impairment, and } \\
\text { unintentional weight loss }\end{array}$ \\
\hline Kittiskulnam et al. [56] & FNIH & HR & $1.69(0.91,3.14)$ & Age, sex, and race \\
\hline Lin et al. [49] & AWGS & HR & $1.94(0.70,5.42)$ & Age, sex \\
\hline \multicolumn{5}{|l|}{ Inpatients } \\
\hline Harimoto et al. [72] & AWGS & OR & $4.02(1.19,13.5)$ & $\begin{array}{l}\text { Recipient age, donor age, recipient sex, recipient status (hospitalized/home), } \\
\text { BMI, DM, MELD score, HCC/non-HCC, major vessel shunt, GV/SLV, portal } \\
\text { vein pressure at laparotomy, and low skeletal muscle area }\end{array}$ \\
\hline Hu et al. [73] & AWGS & HR & $\begin{array}{l}4.25(2.22,8.12)^{\mathrm{g}} \\
1.66(0.48,5.72)^{\mathrm{h}} \\
4.78(2.09,11.0)^{\mathrm{i}}\end{array}$ & Crude \\
\hline Kaido et al. [74] & AWGS & OR & $13.11^{\mathrm{f}}$ & Crude \\
\hline Yang et al. [75] & AWGS & HR & $2.26(1.29,3.95)$ & Age and sex \\
\hline Yoo et al. [76] & AWGS & HR & $1.84(0.69,4.92)$ & Age, sex, BMI, and Koval $(\geq 4)$ \\
\hline Zhang et al. [77] & AWGS & HR & $0.41(0.13,1.33)$ & Age, sex, and CCI \\
\hline Atmis et al. [66] & EWGSOP & HR & $6.41(2.93,14.4)$ & Age, sex, BMI, and ADL \\
\hline Bayraktar et al. [60] & EWGSOP & OR & $3.22^{\mathrm{f}}$ & Crude \\
\hline Beretta et al. [58] & EWGSOP & HR & $1.34(0.52,3.49)$ & Age and sex \\
\hline Bernabeu-Wittel et al. [67] ${ }^{j}$ & EWGSOP & HR & $1.34(0.94,1.91)$ & Age and sex \\
\hline Cerri et al. [63] & EWGSOP & OR & $8.56^{\mathrm{f}}$ & Crude \\
\hline Gariballa et al. [61] & EWGSOP & OR & $3.46^{\mathrm{f}}$ & Crude \\
\hline Isoyama et al. [62] & EWGSOP & HR & $2.94(1.64,5.27)$ & Age and sex \\
\hline Perez-Zepeda et al. [64] & EWGSOP & HR & $2.23(1.15,4.34)$ & Age, sex, and CCI \\
\hline Pourhassan et al. [65] & EWGSOP & OR & $1.67^{\mathrm{f}}$ & Crude \\
\hline Rustani et al. [68] & EWGSOP & OR & $4.58^{\mathrm{f}}$ & Crude \\
\hline Sanchez-Rodriguez et al. [69] & EWGSOP & OR & $0.85(0.44,1.63)$ & $\begin{array}{l}\text { Age, sex, CCI }>2 \text {, unintentional weight loss, malnutrition, overweight-obesity, } \\
\text { nutritional deficiency, and cachexia }\end{array}$ \\
\hline
\end{tabular}


Table 3 (continued)

\begin{tabular}{|c|c|c|c|c|}
\hline Author & $\begin{array}{l}\text { Sarcopenia } \\
\text { definition }\end{array}$ & EM & Effect size $(95 \% \mathrm{CI})$ & Adjustments \\
\hline Sánchez-Rodriguez et al. [24] & EWGSOP & OR & $2.20^{\mathrm{f}}$ & Crude \\
\hline Teng et al. [71] & EWGSOP & OR & $0.87^{\mathrm{f}}$ & Crude \\
\hline Vetrano et al. [59] & EWGSOP & HR & $1.56(1.10,2.30)$ & Age and sex \\
\hline Malafarina et al. [79] & EWGSOP2 & HR & $1.67(1.11,2.51)$ & Age, sex, and dialysis center \\
\hline \multirow[t]{2}{*}{ Bianchi et al. [78] } & EWGSOP2 & HR & $1.87(1.35,2.59)$ & Age and sex \\
\hline & FNIH & HR & $1.54(1.11,2.15)$ & Age and sex \\
\hline Sipers et al. [57] & EWGSOP & HR & $4.31(2.09,8.85)$ & Crude \\
\hline \multicolumn{5}{|l|}{ Nursing home residents } \\
\hline Buckinx et al. [84] & EWGSOP & OR & $1.70(1.10,2.92)$ & $\begin{array}{l}\text { Age, sex, arm circumference, general health perception, emotional role } \\
\text { function, TFI, SHARE-FI, living in nursing homes, TT, and SPPB }\end{array}$ \\
\hline Henwood et al. [82] & EWGSOP & OR & $1.32^{\mathrm{f}}$ & Crude \\
\hline Landi et al. [80] & EWGSOP & HR & $3.19(1.17,8.66)$ & Age and sex \\
\hline Saka et al. [81] & EWGSOP & OR & $2.97^{\mathrm{f}}$ & Crude \\
\hline Yalcin et al. [83] & EWGSOP & $\mathrm{HR}$ & $2.63(1.22,5.65)$ & Age and sex \\
\hline
\end{tabular}

ADL, activities of daily living; ALT, alanine transaminase; AWGS, Asian Working Group for Sarcopenia; CCI, Charlson Comorbidity Index; CIRS, chronic inflammatory response syndrome; COPD, chronic obstructive pulmonary disease; Cr, creatinine; CVD, cardiovascular disease; DM, diabetes mellitus; EM, effect measure; EWGSOP, European Working Group on Sarcopenia in Older People 2010; EWGSOP2, European Working Group on Sarcopenia in Older people 2018; F, Female; FNIH, Foundation for the National Institutes of Health; GDS, Geriatric Depression Scale; GV/SLV, graft volume/standard liver volume; Hb, hemoglobin; HCC, hepatocellular carcinoma; HR, hazard ratio; IADL, instrumental activities of daily living; IHD, ischemic heart disease; IWGS, International Working Group for Sarcopenia; Kt/V, fractional urea clearance; M, Male; MELD, model for end-stage liver disease; MMSE, Mini-Mental State Examination; nPCR, normalized protein catabolic rate; OR, odds ratio; PAH, pulmonary arterial hypertension; SBP, systolic blood pressure; SHAREFI, share frailty instrument; SPPB, short physical performance battery; T4, thyroxine; TFI, Tilburg Frailty Index; TT, Tinetti Test. ${ }^{a}$ Men Study Sleep Study Ancillary Study. ${ }^{\mathrm{b}}$ Health Aging and Body Composition Study. ${ }^{\mathrm{c}}$ Study of Osteoporotic Fractures - Original. ${ }^{\mathrm{d}}$ Study of Osteoporotic Fractures - African American cohorts. ${ }^{\text {e }}$ Framingham Study Offspring cohort. ${ }^{\mathrm{f}}$ Calculated by $2 \times 2$ table. ${ }^{\mathrm{g}}$ Sarcopenia with risk of malnutrition. ${ }^{\mathrm{h}}$ Sarcopenia and normal nutrition. ${ }^{\mathrm{i}}$ Malnutrition-sarcopenia syndrome. ${ }^{\mathrm{j}}$ Outpatients and inpatients.

tifying individuals who are at risk of sarcopenia using screening tools and diagnosing sarcopenia timely is essential to delay adverse health outcomes.

Furthermore, the association between sarcopenia and mortality was independent of the follow-up period. Our finding that the mortality risk is higher in the long term (follow-up period $>24$ months) for inpatients is different from a previous study conducted in acute settings where short-term (in-hospital) mortality risk was higher than long-term (12 months) mortality [59]; however; this could be explained by the differences in cutoffs utilized to define short and long term. The comparison of short- and long-term mortality within populations is limited. Given the heterogeneous nature of inpatient characteristics, further research is warranted to explore the appropriate cutoff for short-term and long-term mortality of patients admitted due to different reasons.

A significant association with mortality was found in both high and low risk of bias articles. High risk of bias articles lack adjustments for confounding effects, which may result in an overestimation of the association between sarcopenia and mortality. As the prevalence of sarcopenia is higher in males and with chronological age $[96,97]$, analyses not adjusted for confounders such as age and sex are therefore likely to have overestimated the association compared to adjusted analyses. A higher pooled HR and OR in 


\begin{tabular}{|c|c|c|c|c|c|c|c|c|c|c|}
\hline \multirow[b]{2}{*}{ Author, year } & \multirow[b]{2}{*}{ Population } & \multirow[b]{2}{*}{$N$} & \multicolumn{5}{|c|}{ Statistics for each study } & & & \multirow[b]{2}{*}{$\begin{array}{l}\text { Relative } \\
\text { weight }\end{array}$} \\
\hline & & & $\begin{array}{l}\text { Hazard } \\
\text { ratio }\end{array}$ & $\begin{array}{l}\text { Lower } \\
\text { limit }\end{array}$ & $\begin{array}{l}\text { Upper } \\
\text { limit }\end{array}$ & $Z$ value & $p$ value & \multicolumn{2}{|c|}{ Hazard ratio and $95 \% \mathrm{Cl}$} & \\
\hline Yuki, 2017 (M) & Community-dwelling & 365 & 1.860 & 1.028 & 3.364 & 2.052 & 0.040 & & & 4.98 \\
\hline Yuki, 2017(F) & Community-dwelling & 355 & 1.030 & 0.409 & 2.594 & 0.063 & 0.950 & & & 2.72 \\
\hline Alexandre, 2014 & Community-dwelling & 1,149 & 1.720 & 1.199 & 2.468 & 2.945 & 0.003 & & & 7.86 \\
\hline Arango-Lopera, 2013 & Community-dwelling & 345 & 2.390 & 1.051 & 5.435 & 2.079 & 0.038 & & & 3.24 \\
\hline Bianchi, 2016 & Community-dwelling & 538 & 2.120 & 1.048 & 4.290 & 2089 & 0.037 & & & 4.01 \\
\hline Brown, 2015 & Community-dwelling & 4,425 & 1.400 & 1.249 & 1.569 & 5.787 & 0.000 & & $=$ & 11.35 \\
\hline Kim, 2014 (M) & Community-dwelling & 284 & 4.630 & 1.616 & 13.266 & 2.853 & 0.004 & & & 2.21 \\
\hline Kim, 2014 (F) & Community-dwelling & 272 & 0860 & 0.182 & 4.059 & -0.190 & 0.849 & & & 1.13 \\
\hline Landi, 2016 & Community-dwelling & 354 & 2.910 & 1.497 & 5.658 & 3.149 & 0.002 & & & 4.33 \\
\hline Costanzo, 2020 & Community-dwelling & 535 & 2.300 & 0.853 & 6.202 & 1.646 & 0.100 & & & 2.43 \\
\hline de Buyser, 2016 & Community-dwelling & 191 & 2.500 & 1.315 & 4.754 & 2.795 & 0.005 & & & 4.52 \\
\hline Hirani, 2015 & Community-dwelling & 1,678 & 1.690 & 1.170 & 2.441 & 2.799 & 0.005 & & & 7.77 \\
\hline McLean, 2014 (M) MrOs & Community-dwelling & 3,006 & 1.270 & 0.653 & 2.471 & 0.704 & 0.481 & & 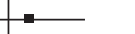 & 4.32 \\
\hline McLean, 2014 (M) HABC & Community-dwelling & 894 & 1.510 & 0.612 & 3.724 & 0895 & 0.371 & & & 2.82 \\
\hline McLean, 2014 (F) H ABC & Community-dwelling & 912 & 1.150 & 0281 & 4.712 & 0.194 & 0.846 & & & 1.34 \\
\hline McLean, 2014(F) SOF-W & Community-dwelling & 403 & 1.650 & 0.519 & 5.243 & 0849 & 0.396 & & & 1.89 \\
\hline McLean, 2014(F) SOF-AA & Community-dwelling & 297 & 3.620 & 0.492 & 26.647 & 1.263 & 0.207 & & & 0.71 \\
\hline McLean, 2014 (F) Fram. & Community-dwelling & 257 & 0600 & 0.079 & 4.530 & -0.495 & 0.620 & & & 0.69 \\
\hline Tang, 2018 & Community-dwelling & 728 & 3.440 & 1.171 & 10.107 & 2.247 & 0.025 & & & 2.12 \\
\hline Kim, 2016 (M) & Community-dwelling & 285 & 1.830 & 0887 & 3.776 & 1.635 & 0.102 & & & 3.87 \\
\hline Kim, 2016 (F) & Community-dwelling & 275 & 0980 & 0.272 & 3.528 & -0.031 & 0.975 & & & 1.59 \\
\hline Bachettini, 2019 & Community-dwelling & 1,291 & 1.180 & 0528 & 2.639 & 0.403 & 0.687 & & - & 3.34 \\
\hline Sim, 2019 & Community-dwelling & 903 & 1.880 & 1.240 & 2.850 & 2.973 & 0.003 & & & 7.07 \\
\hline Sobestiansky, 2019 & Community-dwelling & 287 & 1.950 & 1.119 & 3.398 & 2.357 & 0.018 & & & 5.36 \\
\hline Cawthon, 2015 & Community-dwelling & 5,934 & 3.490 & 2.012 & 6.055 & 4.446 & 0.000 & & & 5.41 \\
\hline \multirow[t]{2}{*}{ Locquet, 2019} & Community-dwelling & 534 & 4200 & 1.741 & 10.134 & 3.193 & 0.001 & & & 2.92 \\
\hline & & & 1.888 & 1.587 & 2.245 & 7.188 & 0.000 & & & \\
\hline Mori, 2019 & Outpatients & 308 & 1.310 & 0.814 & 2.109 & 1.111 & 0.267 & & & 24.92 \\
\hline Giglio, 2018 & Outpatients & 170 & 2.090 & 1.045 & 4.180 & 2.084 & 0.037 & & & 16.28 \\
\hline Olesen, 2019 & Outpatients & 182 & 6.690 & 1.794 & 24.952 & 2.830 & 0.005 & & & 6.01 \\
\hline Aliberti, 2019 & Outpatients & 665 & 1.690 & 1.048 & 2.725 & 2.153 & 0.031 & & & 24.85 \\
\hline Kittiskulnam, 2017 & Outpatients & 645 & 1.690 & 0.910 & 3.139 & 1.661 & 0.097 & & & 18.77 \\
\hline \multirow[t]{2}{*}{ Lin, 2019} & Outpatients & 126 & 1.940 & 0.697 & 5.398 & 1.269 & 0.204 & & & 9.18 \\
\hline & & & 1.806 & 1.279 & 2.551 & 3.356 & 0.001 & & & \\
\hline $\mathrm{Hu}, 2017$ (MN) & Inpatients & 253 & 4.250 & 2.222 & 8.128 & 4.374 & 0.000 & & & 5.85 \\
\hline $\mathrm{Hu}, 2017$ (NN) & Inpatients & 219 & 1.660 & 0.481 & 5.730 & 0802 & 0.423 & & & 2.20 \\
\hline $\mathrm{Hu}, 2017$ (MSS) & Inpatients & 227 & 4.780 & 2.086 & 10.951 & 3.699 & 0.000 & & & 4.19 \\
\hline Yang, 2017 & Inpatients & 288 & 2.260 & 1.292 & 3.955 & 2.856 & 0.004 & & & 6.97 \\
\hline Yoo, 2018 & Inpatients & 324 & 2.260 & 1.292 & 3.955 & 2.856 & 0.004 & & & 6.97 \\
\hline Zhang, 2019 & Inpatients & 345 & 0.410 & 0.128 & 1.311 & -1.503 & 0.133 & & - & 2.45 \\
\hline Atmis, 2019 & Inpatients & 350 & 6.410 & 2.891 & 14.210 & 4.574 & 0.000 & & & 4.44 \\
\hline Beretta, 2020 & Inpatients & 610 & 1.340 & 0.517 & 3.471 & 0.603 & 0.547 & & & 3.40 \\
\hline Bernabeu-Wittel, 2019 & Inpatients & 444 & 1.340 & 0.940 & 1.910 & 1.618 & 0.106 & & - & 10.43 \\
\hline Isoyama, 2014 & Inpatients & 200 & 2.940 & 1.640 & 5270 & 3.621 & 0.000 & & & 6.64 \\
\hline Perez-Zepeda, 2017 & Inpatients & 610 & 2.230 & 1.148 & 4.332 & 2.367 & 0.018 & & & 5.68 \\
\hline Vetrano, 2014 & Inpatients & 770 & 1.560 & 1.079 & 2.256 & 2.363 & 0.018 & & $\rightarrow$ & 10.15 \\
\hline Zengarini, 2019 & Inpatients & 624 & 2.020 & 0983 & 4.152 & 1.913 & 0.056 & & & 5.11 \\
\hline Malafarina, 2019 & Inpatients & 187 & 1.670 & 1.111 & 2.511 & 2.464 & 0.014 & & $\longrightarrow$ & 9.41 \\
\hline Bianchi, 2019 & Inpatients & 610 & 1.870 & 1.350 & 2.590 & 3.766 & 0.000 & & & 11.00 \\
\hline \multirow[t]{2}{*}{ Sipers, 2019} & Inpatients & 81 & 4.310 & 2.094 & 8869 & 3.968 & 0.000 & & & 5.09 \\
\hline & & & 2.151 & 1.764 & 2.623 & 7.570 & 0.000 & & & \\
\hline Landi, 2012 & Nursing home residents & 5122 & 3.190 & 1.173 & 8679 & 2.272 & 0.023 & & & 40.10 \\
\hline \multirow[t]{4}{*}{ Yalcin, 2017} & Nursing home residents & 141 & 2.630 & 1.222 & 5.660 & 2.473 & 0.013 & & & 59.90 \\
\hline & & & 2.842 & 1.399 & 5.773 & 2.888 & 0.004 & & & \\
\hline & Overall & & 2.003 & 1.711 & 2.344 & 8.654 & 0.000 & & & \\
\hline & & & & & & & & 0.1 & 1 & \\
\hline
\end{tabular}

Fig. 2. Meta-analysis of the association between sarcopenia and mortality presented in HRs, stratified by population. Heterogeneity $\left(I^{2}\right)$ : community-dwelling adults $(32.4 \%)$, outpatients $(12.4 \%)$, inpatients $(62.1 \%)$, and nursing home residents (0\%). HR, hazard ratio, M, males; F, females; MrOs, Men Study Sleep Study Ancillary Study; HABC, Health Aging and Body Composition Study; SOF-
W, Study of Osteoporotic Fractures - Original; SOF-AA, Study of Osteoporotic Fractures - African American cohorts; Fram., Framingham Study Offspring cohort; MN, sarcopenia with a risk of malnutrition; NN, sarcopenia with normal nutrition; MSS, malnutrition-sarcopenia syndrome. 


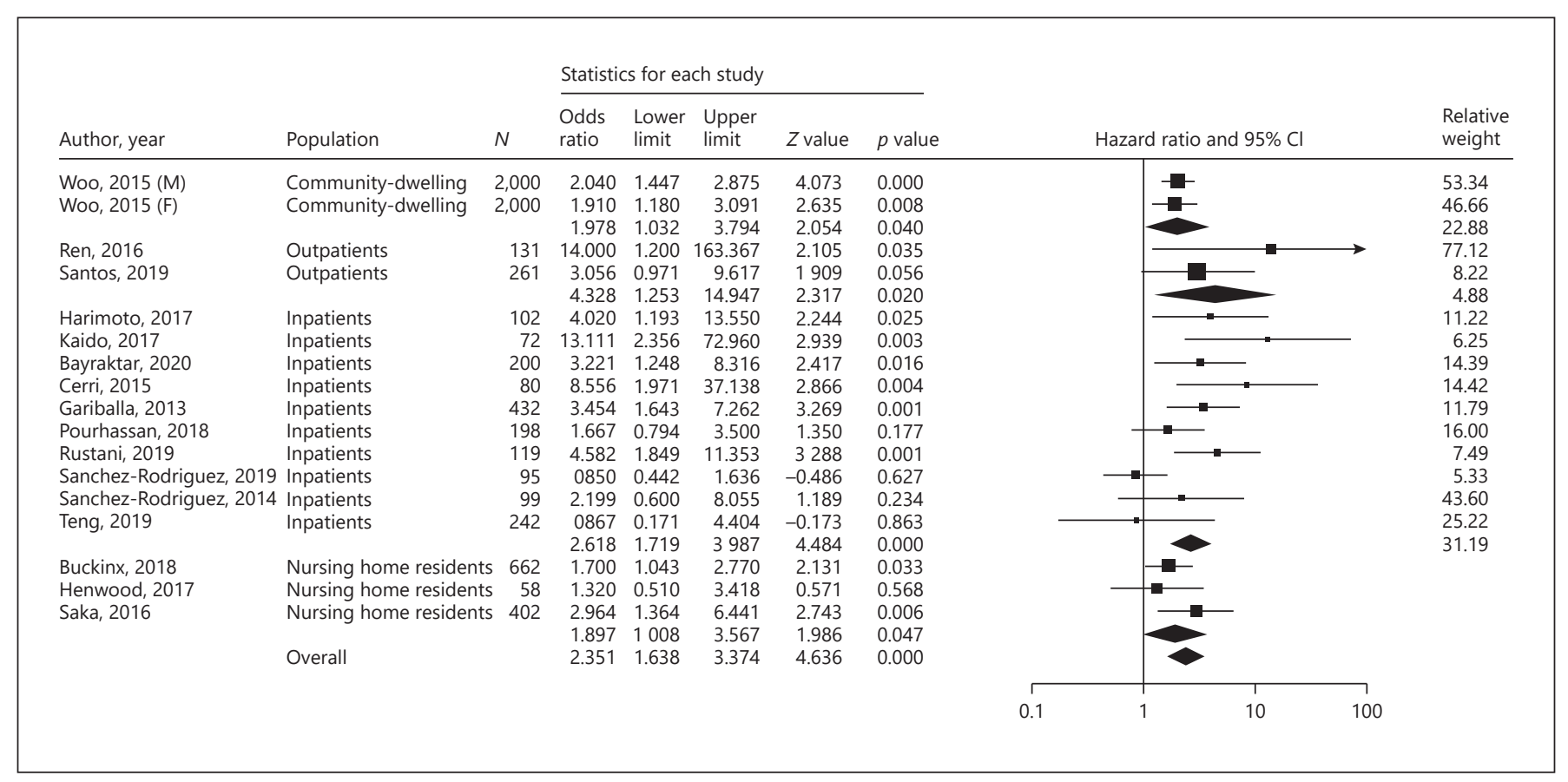

Fig. 3. Meta-analysis of the association between sarcopenia and mortality presented in ORs, stratified by population. Heterogeneity $\left(I^{2}\right)$ : community-dwelling adults (0\%), outpatients (17.4\%), inpatients (60.3\%), and nursing home residents $(0.7 \%)$. OR, odds ratios; $\mathrm{M}$, males; $\mathrm{F}$, females.

high risk of bias articles is hence observed compared to low risk of bias articles, although the heterogeneity of effect estimates was only significantly different for the pooled OR.

Low to moderate heterogeneity was found across all populations, definitions, follow-up periods, and risk of bias groups apart from the pooled FNIH HR in inpatients, where the heterogeneity was high. The high heterogeneity observed in the FNIH subgroup can be explained by the inclusion of both a crude and an adjusted HR in subgroups $[57,78]$.

\section{Strengths and Limitations}

This is the first systematic review and meta-analysis analyzing the association between sarcopenia and mortality within various populations, stratified by the latest working group definitions of sarcopenia: EWGSOP, EWGSOP2, AWGS, and FNIH. Due to the variation in the number of articles included within each population, subgroup analyses were not performed for nursing home residents and individuals with specific diseases such as cancer or renal failure, limiting the generalizability of our results. Furthermore, muscle mass was frequently measured by bioelectrical impedance analysis, which might lead to over-/underestimation of lean mass.

Sarcopenia and Mortality

\section{Conclusion}

Sarcopenia is associated with a significantly higher risk of mortality, independent of population, sarcopenia definition, follow-up period, and risk of bias. This stresses the need for early detection and diagnosis of sarcopenia in all populations to implement interventions preventing and treating sarcopenia in a timely manner.

\section{Acknowledgements}

The authors would like to thank Patrick Condron (Senior Liaison Librarian, Brownless Biomedical Library, Faculty of Medicine, Dentistry \& Health Sciences, The University of Melbourne), who greatly assisted with the construction of the search strategy.

\section{Statement of Ethics}

Ethical approval was not required.

\section{Conflict of Interest Statement}

J.X., C.S.W., K.K., E.M.R., and A.B.M. declare they have no conflicts of interest. 


\section{Funding Sources}

Unrestricted funding was provided by the University of Melbourne to A.B.M.

\section{Author Contributions}

J.X.: conceptualization, methodology, investigation, data curation, formal analysis, and writing - original draft. C.S.W.: conceptualization, methodology, investigation, data curation, supervision, and writing - review and editing. K.K.: conceptualization, methodology, investigation, data curation, and writing - review and editing. E.M.R.: conceptualization, methodology, investigation, supervision, and writing - review and editing. A.B.M.: conceptualization, methodology, investigation, supervision, and writing - review and editing.

\section{References}

1 Makizako H, Nakai Y, Tomioka K, Taniguchi Y. Prevalence of sarcopenia defined using the Asia Working Group for Sarcopenia criteria in Japanese community-dwelling older adults: a systematic review and meta-analysis. Phys Ther Res. 2019;22(2):53-7.

2 Mayhew AJ, Amog K, Phillips S, Parise G, McNicholas PD, de Souza RJ, et al. The prevalence of sarcopenia in community-dwelling older adults, an exploration of differences between studies and within definitions: a systematic review and meta-analyses. Age Ageing. 2019;48(1):48-56.

3 Reijnierse EM, Trappenburg MC, Leter MJ, Blauw GJ, Sipilä S, Sillanpää E, et al. The impact of different diagnostic criteria on the prevalence of sarcopenia in healthy elderly participants and geriatric outpatients. Gerontology. 2015;61(6):491-6.

4 Churilov I, Churilov L, MacIsaac RJ, Ekinci EI. Systematic review and meta-analysis of prevalence of sarcopenia in post acute inpatient rehabilitation. Osteoporos Int. 2018; 29(4):805-12.

5 Pacifico J, Geerlings MAJ, Reijnierse EM, Phassouliotis C, Lim WK, Maier AB. Prevalence of sarcopenia as a comorbid disease: a systematic review and meta-analysis. Exp Gerontol. 2020;131:110801.

6 Chen LK, Woo J, Assantachai P, Auyeung TW, Chou MY, Iijima K, et al. Asian Working Group for Sarcopenia: 2019 consensus update on sarcopenia diagnosis and treatment. J Am Med Dir Assoc. 2020;21(3):300-e2.

7 Cruz-Jentoft AJ, Bahat G, Bauer J, Boirie Y, Bruyère $\mathrm{O}$, Cederholm $\mathrm{T}$, et al. Sarcopenia: revised European consensus on definition and diagnosis. Age Ageing. 2019;48(1):601-31.

8 Studenski SA, Peters KW, Alley DE, Cawthon PM, McLean RR, Harris TB, et al. The FNIH sarcopenia project: rationale, study description, conference recommendations, and final estimates. J Gerontol A Biol Sci Med Sci. 2014; 69(5):547-58.

9 Chen LK, Liu LK, Woo J, Assantachai P, Auyeung TW, Bahyah KS, et al. Sarcopenia in Asia: consensus report of the Asian Working Group for sarcopenia. J Am Med Dir Assoc. 2014;15(2):95-101.
10 Fielding RA, Vellas B, Evans WJ, Bhasin S, Morley JE, Newman AB, et al. Sarcopenia: an undiagnosed condition in older adults. Current consensus definition: prevalence, etiology, and consequences. International working group on sarcopenia. J Am Med Dir Assoc. 2011;12(4):249-56.

11 Cruz-Jentoft AJ, Baeyens JP, Bauer JM, Boirie Y, Cederholm T, Landi F, et al. Sarcopenia: European consensus on definition and diagnosis: report of the European Working Group on sarcopenia in older people. Age Ageing. 2010;39(4):412-23.

12 Yeung SSY, Reijnierse EM, Pham VK, Trappenburg MC, Lim WK, Meskers CGM, et al. Sarcopenia and its association with falls and fractures in older adults: a systematic review and meta-analysis. J Cachexia Sarcopenia Muscle. 2019;10(3):485-500.

13 Wang DXM, Yao J, Zirek Y, Reijnierse EM, Maier AB. Muscle mass, strength, and physical performance predicting activities of daily living: a meta-analysis. J Cachexia Sarcopenia Muscle. 2020;11(1):3-25.

14 Beaudart C, Zaaria M, Pasleau F, Reginster JY, Bruyère $\mathrm{O}$. Health outcomes of sarcopenia: a systematic review and meta-analysis. PLoS One. 2017;12(1):e0169548-e48.

15 Liu P, Hao Q, Hai S, Wang H, Cao L, Dong B. Sarcopenia as a predictor of all-cause mortality among community-dwelling older people: a systematic review and meta-analysis. Maturitas. 2017;103:16-22.

16 Zhang X, Wang C, Dou Q, Zhang W, Yang Y, $\mathrm{Xie} \mathrm{X}$. Sarcopenia as a predictor of all-cause mortality among older nursing home residents: a systematic review and meta-analysis. BMJ Open. 2018;8(11):e021252.

17 Mintziras I, Miligkos M, Wächter S, Manoharan J, Maurer E, Bartsch DK. Sarcopenia and sarcopenic obesity are significantly associated with poorer overall survival in patients with pancreatic cancer: systematic review and meta-analysis. Int J Surg. 2018;59:19-26.

18 Chang SF, Lin PL. Systematic literature review and meta-analysis of the association of sarcopenia with mortality. Worldviews Evid Based Nurs. 2016;13(2):153-62.
19 Bhasin S, Travison TG, Manini TM, Patel S, Pencina KM, Fielding RA, et al. Sarcopenia definition: the position statements of the sarcopenia definition and outcomes consortium. J Am Geriatr Soc. 2020;68(7):1410-8.

20 Bijlsma AY, Meskers CG, Ling CH, Narici M, Kurrle SE, Cameron ID, et al. Defining sarcopenia: the impact of different diagnostic criteria on the prevalence of sarcopenia in a large middle aged cohort. Age. 2013;35(3):871-81.

21 Van Ancum JM, Alcazar J, Meskers CGM, Nielsen BR, Suetta C, Maier AB. Impact of using the updated EWGSOP2 definition in diagnosing sarcopenia: a clinical perspective. Arch Gerontol Geriatr. 2020;90:104125.

22 Moher D, Liberati A, Tetzlaff J, Altman DG. Preferred reporting items for systematic reviews and meta-analyses: the PRISMA statement. BMJ. 2009;339:b2535.

23 Sánchez-Rodríguez D, Marco E, Annweiler C, Ronquillo-Moreno N, Tortosa A, VázquezIbar O, et al. Malnutrition in postacute geriatric care: basic ESPEN diagnosis and etiology based diagnoses analyzed by length of stay, inhospital mortality, and functional rehabilitation indexes. Arch Gerontol Geriatr. 2017;73: 169-76.

24 Sánchez-Rodríguez D, Marco E, Miralles R, Fayos M, Mojal S, Alvarado M, et al. Sarcopenia, physical rehabilitation and functional outcomes of patients in a subacute geriatric care unit. Arch Gerontol Geriatr. 2014;59(1): 39-43.

25 Landi F, Cruz-Jentoft AJ, Liperoti R, Russo A, Giovannini S, Tosato M, et al. Sarcopenia and mortality risk in frail older persons aged 80 years and older: results from ilSIRENTE study. Age Ageing. 2013;42(2):203-9.

26 Landi F, Calvani R, Tosato M, Martone AM, Bernabei R, Onder G, et al. Impact of physical function impairment and multimorbidity on mortality among community-living older persons with sarcopaenia: results from the ilSIRENTE prospective cohort study. BMJ Open. 2016;6(7):e008281.

27 Lo CK, Mertz D, Loeb M. Newcastle-Ottawa scale: comparing reviewers' to authors' assessments. BMC Med Res Methodol. 2014;14: 45. 
28 Higgins JP, Thompson SG. Quantifying heterogeneity in a meta-analysis. Stat Med. 2002; 21(11):1539-58.

29 Egger M, Davey Smith G, Schneider M, Minder C. Bias in meta-analysis detected by a simple, graphical test. BMJ. 1997;315(7109):62934.

30 Arango-Lopera VE, Arroyo P, Gutiérrez-Robledo LM, Pérez-Zepeda MU, Cesari M. Mortality as an adverse outcome of sarcopenia. J Nutr Health Aging. 2013;17(3):259-62.

31 Alexandre Tda S, Duarte YA, Santos JL, Wong R, Lebrão ML. Sarcopenia according to the European Working Group on sarcopenia in older people (EWGSOP) versus dynapenia as a risk factor for mortality in the elderly. J Nutr Health Aging. 2014;18(8):751-6.

32 Kim JH, Lim S, Choi SH, Kim KM, Yoon JW, Kim KW, et al. Sarcopenia: an independent predictor of mortality in community-dwelling older Korean men. J Gerontol A Biol Sci Med Sci. 2014;69(10):1244-52.

33 Cawthon PM, Blackwell TL, Cauley J, Kado DM, Barrett-Connor E, Lee CG, et al. Evaluation of the usefulness of consensus definitions of sarcopenia in older men: results from the observational osteoporotic fractures in Men Cohort Study. J Am Geriatr Soc. 2015;63(11) 2247-59.

34 Woo J, Leung J, Morley JE. Defining sarcopenia in terms of incident adverse outcomes. J Am Med Dir Assoc. 2015;16(3):247-52.

35 Bianchi L, Ferrucci L, Cherubini A, Maggio M, Bandinelli S, Savino E, et al. The predictive value of the EWGSOP definition of sarcopenia: results from the InCHIANTI Study. J Gerontol A Biol Sci Med Sci. 2016;71(2):25964.

36 Brown JC, Harhay MO, Harhay MN. Sarcopenia and mortality among a populationbased sample of community-dwelling older adults. J Cachexia Sarcopenia Muscle. 2016; 7(3):290-8.

37 Locquet M, Beaudart C, Hajaoui M, Petermans J, Reginster JY, Bruyère O. Three-year adverse health consequences of sarcopenia in community-dwelling older adults according to 5 diagnosis definitions. J Am Med Dir Assoc. 2019;20(1):43-e2.

38 Sim M, Prince RL, Scott D, Daly RM, Duque G, Inderjeeth CA, et al. Sarcopenia definitions and their associations with mortality in older Australian women. J Am Med Dir Assoc. 2019;20(1):76-e2.

39 Sobestiansky S, Michaelsson K, Cederholm T. Sarcopenia prevalence and associations with mortality and hospitalisation by various sarcopenia definitions in 85-89 year old community-dwelling men: a report from the ULSAM study. BMC Geriatrics. 2019;19(1):318.

40 Bachettini NP, Bielemann RM, Barbosa-Silva TG, Menezes AMB, Tomasi E, Gonzalez MC. Sarcopenia as a mortality predictor in community-dwelling older adults: a comparison of the diagnostic criteria of the European Working Group on sarcopenia in older people. Eur J Clin Nutr. 2020;74(4):573-80.
41 McLean RR, Shardell MD, Alley DE, Cawthon PM, Fragala MS, Harris TB, et al. Criteria for clinically relevant weakness and low lean mass and their longitudinal association with incident mobility impairment and mortality: the foundation for the National Institutes of Health (FNIH) sarcopenia project. J Gerontol A Biol Sci Med Sci. 2014;69(5):576-83.

42 Hirani V, Blyth F, Naganathan V, Le Couteur DG, Seibel MJ, Waite LM, et al. Sarcopenia is associated with incident disability, institutionalization, and mortality in communitydwelling older men: the concord health and ageing in men project. J Am Med Dir Assoc. 2015; 16(7):607-13

43 De Buyser SL, Petrovic M, Taes YE, Toye KR, Kaufman JM, Lapauw B, et al. Validation of the FNIH sarcopenia criteria and SOF frailty index as predictors of long-term mortality in ambulatory older men. Age Ageing. 2016; 45(5):602-8.

44 Moon JH, Kim KM, Kim JH, Moon JH, Choi $\mathrm{SH}$, Lim S, et al. Predictive values of the new sarcopenia index by the foundation for the national institutes of health sarcopenia project for mortality among older Korean adults. PLoS One. 2016;11(11):e0166344.

45 Tang TC, Hwang AC, Liu LK, Lee WJ, Chen $\mathrm{LY}, \mathrm{Wu} \mathrm{YH}$, et al. FNIH-defined sarcopenia predicts adverse outcomes among community-dwelling older people in Taiwan: results from I-Lan Longitudinal Aging Study. J Gerontol A Biol Sci Med Sci. 2018;73(6):82834.

46 Yuki A, Ando F, Otsuka R, Shimokata H. Response to the letter of Dr Kizilarslanoglu, "Sarcopenia based on the Asian Working Group for sarcopenia criteria and all-cause mortality risk in older Japanese adults". Geri atr Gerontol Int. 2017;17(10):1762-3.

47 Costanzo L, De Vincentis A, Di Iorio A, Bandinelli S, Ferrucci L, Antonelli Incalzi R, et al. Impact of low muscle mass and low muscle strength according to EWGSOP2 and EWGSOP1 in community-dwelling older people. J Gerontol A Biol Sci Med Sci. 2020;75(7): 1324-30.

48 Giglio J, Kamimura MA, Lamarca F, Rodrigues J, Santin F, Avesani CM. Association of sarcopenia with nutritional parameters, quality of life, hospitalization, and mortality rates of elderly patients on hemodialysis. J Ren Nutr. 2018;28(3):197-207.

49 Lin YL, Liou HH, Wang CH, Lai YH, Kuo CH, Chen SY, et al. Impact of sarcopenia and its diagnostic criteria on hospitalization and mortality in chronic hemodialysis patients: a 3-year longitudinal study. J Formos Med Assoc. 2020;119(7):1219-29.

50 Olesen SS, Büyükuslu A, Køhler M, Rasmussen HH, Drewes AM. Sarcopenia associates with increased hospitalization rates and reduced survival in patients with chronic pancreatitis. Pancreatology. 2019;19(2):245-51.
51 Santos LAA, Lima TB, Ietsugu MV, Nunes HRC, Qi X, Romeiro FG. Anthropometric measures associated with sarcopenia in outpatients with liver cirrhosis. Nutr Diet. 2019; 76(5):613-9.

52 Ren H, Gong D, Jia F, Xu B, Liu Z. Sarcopenia in patients undergoing maintenance hemodialysis: incidence rate, risk factors and its effect on survival risk. Ren Fail. 2016;38(3):364-71.

53 Kamijo Y, Kanda E, Ishibashi Y, Yoshida M. Sarcopenia and frailty in PD: impact on mortality, malnutrition, and inflammation. Perit Dial Int. 2018;38(6):447-54.

54 Mori K, Nishide K, Okuno S, Shoji T, Emoto $\mathrm{M}$, Tsuda A, et al. Impact of diabetes on sarcopenia and mortality in patients undergoing hemodialysis. BMC Nephrol. 2019;20(1): 105-5.

55 Aliberti MJR, Szlejf C, Covinsky KE, Lee SJ, Jacob-Filho W, Suemoto CK, et al. Prognostic value of a rapid sarcopenia measure in acutely ill older adults. Clin Nutr. 2020;39(7):211420.

56 Kittiskulnam P, Chertow GM, Carrero JJ, Delgado C, Kaysen GA, Johansen KL. Sarcopenia and its individual criteria are associated, in part, with mortality among patients on hemodialysis. Kidney Int. 2017;92(1):238-47.

57 Sipers WMWH, de Blois W, Schols JMGA, van Loon LJC, Verdijk LB. Sarcopenia is related to mortality in the acutely hospitalized geriatric patient. J Nutr Health Aging. 2019; 23(2):128-37.

58 Beretta MV, Dantas Filho FF, Freiberg RE, Feldman JV, Nery C, Rodrigues TC. Sarcopenia and type 2 diabetes mellitus as predictors of 2-year mortality after hospital discharge in a cohort of hospitalized older adults. Diabetes Res Clin Pract. 2020;159:107969.

59 Vetrano DL, Landi F, Volpato S, Corsonello A, Meloni E, Bernabei R, et al. Association of sarcopenia with short- and long-term mortality in older adults admitted to acute care wards: results from the CRIME study. Gerontol A Biol Sci Med Sci. 2014;69(9): 1154-61.

60 Bayraktar E, Tasar PT, Binici DN, Karasahin O, Timur O, Sahin S. Relationship between sarcopenia and mortality in elderly inpatients. Eurasian J Med. 2020;52(1):29-33.

61 Gariballa S, AlessaSarcopenia A. prevalence and prognostic significance in hospitalized patients. Clin Nutr. 2013;32(5):772-6.

62 Isoyama N, Qureshi AR, Avesani CM, et al Comparative associations of muscle mass and muscle strength with mortality in dialysis patients. Clin J Am Soc Nephrol. 2014;9(10): $1720-8$.

63 Cerri AP, Bellelli G, Mazzone A, et al. Sarcopenia and malnutrition in acutely ill hospitalized elderly: Prevalence and outcomes. Clin Nutr. 2015;34(4):745-51.

64 Pérez-Zepeda MU, Sgaravatti A, Dent E. Sarcopenia and post-hospital outcomes in older adults: A longitudinal study. Arch Gerontol Geriatr. 2017;69:105-9. 
65 Pourhassan M, Norman K, Müller MJ, et al. Impact of Sarcopenia on One-Year Mortality Among Older Hospitalized Patients with Impaired Mobility. J Frailty Aging. 2018;7(1): $40-6$.

66 Atmis V, Yalcin A, Silay K, et al. The relationship between all-cause mortality sarcopenia and sarcopenic obesity among hospitalized older people. Aging Clin Exp Res. 2019; 31(11):1563-72.

67 Bernabeu-Wittel M, González-Molina Á, Fernández-Ojeda R, et al. Impact of sarcopenia and frailty in a multicenter cohort of polypathological patients. Clin Med. 2019; 8(4):535.

68 Rustani K, Kundisova L, Capecchi PL, et al. Prevalence of sarcopenia and its impact on mortality and readmission rates amongst geriatric patients. Arch Gerontol Geriatr. 2019; 67(4):200-6.

69 Sánchez-Rodríguez D, Annweiler C, Ronquillo-Moreno N, et al. Prognostic value of the ESPEN consensus and guidelines for malnutrition: prediction of post-discharge clinical outcomes in older inpatients. Nutr Clin Pract. 2019;34(2):304-12.

70 Zengarini E, Giacconi R, Mancinelli L, et al. Prognosis and interplay of cognitive impairment and sarcopenia in older adults discharged from acute care hospitals. J Clin Med. 2019;8(10):1693.

71 Teng CH, Chen SY, Wei YC, et al. Effects of sarcopenia on functional improvement over the first year after cardiac surgery: a cohort study. Eur J Cardiovasc Nurs. 2019;18(4): 309-17.

72 Harimoto N, Yoshizumi T, Izumi T, Motomura T, Harada N, Itoh S, et al. Clinical outcomes of living liver transplantation according to the presence of sarcopenia as defined by skeletal muscle mass, hand grip, and gait speed. Transplant Proc. 2017;49(9):2144-52.

$73 \mathrm{Hu}$ X, Zhang L, Wang H, Hao Q, Dong B, Yang M. Malnutrition-sarcopenia syndrome predicts mortality in hospitalized older patients. Sci Rep. 2017;7(1):3171.

74 Kaido T, Tamai Y, Hamaguchi Y, Okumura S, Kobayashi A, Shirai H, et al. Effects of pretransplant sarcopenia and sequential changes in sarcopenic parameters after living donor liver transplantation. Nutrition. 2017;33:1958.

75 Yang $\mathrm{M}$, Hu X, Wang H, Zhang L, Hao Q, Dong B. Sarcopenia predicts readmission and mortality in elderly patients in acute care wards: a prospective study. J Cachexia Sarcopenia Muscle. 2017;8(2):251-8.
76 Yoo JI, Kim H, Ha YC, Kwon HB, Koo KH. Osteosarcopenia in patients with hip fracture is related with high mortality. J Korean Med Sci. 2018;33(4):e27.

77 Zhang N, Zhu WL, Liu XH, Chen W, Zhu ML, Kang L, et al. Prevalence and prognostic implications of sarcopenia in older patients with coronary heart disease. J Geriatr Cardiol. 2019;16(10):756-63.

78 Bianchi L, Maietti E, Abete P, Bellelli G, Bo M, Cherubini A, et al. Comparing EWGSOP2 and FNIH sarcopenia definitions: agreement and three-year survival prognostic value in older hospitalized adults. The GLISTEN Study. J Gerontol A Biol Sci Med Sci. 2020; 75(7):1331-7.

79 Malafarina V, Malafarina C, Biain Ugarte A, Martinez JA, Abete Goñi I, Zulet MA. Factors asssociated with sarcopenia and 7-year mortality in very old patients with hip fracture admitted to rehabilitation units: a Pragmatic Study. Nutrients. 2019;11(9):2243.

80 Landi F, Liperoti R, Fusco D, Mastropaolo S, Quattrociocchi D, Proia A, et al. Sarcopenia and mortality among older nursing home residents. J Am Med Dir Assoc. 2012;13(2):1216.

81 Saka B, Ozkaya H, Karisik E, Akin S, Akpinar TS, Tufan F, et al. Malnutrition and sarcopenia are associated with increased mortality rate in nursing home residents: a prospective study. Eur Geriatr Med. 2016;7(3):232-8.

82 Henwood T, Hassan B, Swinton P, Senior H, Keogh J. Consequences of sarcopenia among nursing home residents at long-term followup. Geriatr Nurs. 2017;38(5):406-11.

83 Yalcin A, Aras S, Atmis V, Cengiz OK, Cinar E, Atli T, et al. Sarcopenia and mortality in older people living in a nursing home in Turkey. Geriatr Gerontol Int. 2017;17(7):111824.

84 Buckinx F, Croisier JL, Reginster JY, Lenaerts $\mathrm{C}$, Brunois T, Rygaert X, et al. Prediction of the incidence of falls and deaths among elderly nursing home residents: the SENIOR Study. J Am Med Dir Assoc. 2018;19(1):1824.

85 Greco EA, Pietschmann P, Migliaccio S. Osteoporosis and sarcopenia increase frailty syndrome in the elderly. Front Endocrinol. 2019;10:255-5.

86 Dufour AB, Hannan MT, Murabito JM, Kiel DP, McLean RR. Sarcopenia definitions considering body size and fat mass are associated with mobility limitations: the Framingham Study. J Gerontol A Biol Sci Med Sci. 2013; 68(2):168-74
87 Alley DE, Koster A, Mackey D, Cawthon P, Ferrucci L, Simonsick EM, et al. Hospitalization and change in body composition and strength in a population-based cohort of older persons. J Am Geriatr Soc. 2010;58(11): 2085-91.

88 Bijlsma AY, Pasma JH, Lambers D, Stijntjes M, Blauw GJ, Meskers CG, et al. Muscle strength rather than muscle mass is associated with standing balance in elderly outpatients. J Am Med Dir Assoc. 2013;14(7):493-8.

89 Muir SW, Berg K, Chesworth B, Klar N, Speechley M. Quantifying the magnitude of risk for balance impairment on falls in community-dwelling older adults: a systematic review and meta-analysis. J Clin Epidemiol. 2010;63(4):389-406.

90 Gingrich A, Volkert D, Kiesswetter E, Thomanek M, Bach S, Sieber CC, et al. Prevalence and overlap of sarcopenia, frailty, cachexia and malnutrition in older medical inpatients. BMC Geriatr. 2019;19(1):120.

91 Beaudart C, Sanchez-Rodriguez D, Locquet $\mathrm{M}$, Reginster JY, Lengelé L, Bruyère $\mathrm{O}$. Malnutrition as a strong predictor of the onset of sarcopenia. Nutrients. 2019;11(12):2883.

92 Wang YJ, Wang Y, Zhan JK, Tang ZY, He JY, Tan P, et al. Sarco-osteoporosis: prevalence and association with frailty in Chinese community-dwelling older adults. Int J Endocrinol. 2015;2015:482940.

93 Van Ancum JM, Scheerman K, Jonkman NH, Smeenk HE, Kruizinga RC, Meskers CGM, et al. Change in muscle strength and muscle mass in older hospitalized patients: a systematic review and meta-analysis. Exp Gerontol. 2017;92:34-41.

94 Gong G, Wan W, Zhang X, Liu Y, Liu X, Yin J. Correlation between the Charlson comorbidity index and skeletal muscle mass/physical performance in hospitalized older people potentially suffering from sarcopenia. BMC Geriatr. 2019;19(1):367.

95 Reijnierse EM, Buljan A, Tuttle CSL, van Ancum J, Verlaan S, Meskers CGM, et al. Prevalence of sarcopenia in inpatients 70 years and older using different diagnostic criteria. Nurs Open. 2018;6(2):377-83.

96 Cruz-Jentoft AJ, Landi F, Schneider SM, Zúñiga C, Arai H, Boirie Y, et al. Prevalence of and interventions for sarcopenia in ageing adults: a systematic review. Report of the International Sarcopenia Initiative (EWGSOP and IWGS). Age Ageing. 2014;43(6):748-59.

97 Du Y, Wang X, Xie H, Zheng S, Wu X, Zhu X, et al. Sex differences in the prevalence and adverse outcomes of sarcopenia and sarcopenic obesity in community dwelling elderly in East China using the AWGS criteria. BMC Endocr Disord. 2019;19(1):109. 